\title{
Design of a Human Reliability Assessment model for structural engineering
}

\author{
J. de Haan \& K.C. Terwel \\ Department of Structural and Building Engineering, Technical University Delft, The Netherlands \\ S.H.S. Al-Jibouri \\ Department of Construction Management and Engineering, University of Twente, The Netherlands
}

\begin{abstract}
It is generally accepted that humans are the "weakest link" in structural design and construction processes. Despite this, few models are available to quantify human error within engineering processes. This paper demonstrates the use of a quantitative Human Reliability Assessment model within structural engineering. The model consists of four steps. In the first step the process and its boundary conditions are identified. In the second step, the effect of human error on a single task is defined. In the third step, these effects are combined into an overall effect on the engineered structure. Finally, the structural failure probability is estimated by combining the strength and loading conditions by means of a Monte Carlo simulation. The use of the model is demonstrated using a case study based on the design process of a frame structure. This case study shows that the model has the potential to quantify the effect of human errors within carefully defined boundary conditions.
\end{abstract}

\section{INTRODUCTION}

In recent years major structural failure incidents have occurred in the Netherlands. Examples are the collapse of five balconies of a residential building in Maastricht in 2003 and the partial collapse of the roof structure of a football stadium in Enschede in 2011. An important aspect is the presence of human error in both examples, which is far from coincidence. Researchers such as Ellingwood (1987), Kaminetzky (1991), Stewart (1993), Fruhwald et al. (2007) and Vrouwenvelder (2011) have all concluded that most of the structural failures are caused by human errors. However current codes do not take these into account in the determination of the level of safety.

The objective of this paper is to demonstrate the use of quantitative risk analysis models for evaluating the effects of human error in structural engineering. For this a Human Reliability Assessment (HRA) model is proposed and subsequently demonstrated by means of a case study. The proposed HRA model is a Risk Management Support tool aimed towards evaluating low probability-high consequence risks, occurring due to human malfunction in (structural) engineering processes. The model enhances the possibilities for a company to allow the evaluation of the present and future vulnerabilities to catastrophic events.

\section{THEORIES USED FOR THE MODEL}

Error is defined in this study as: "Any member of a set of human actions or activities that exceeds some limit of acceptability" (Kirwan 1994). There are three major elements in the production of an error according to (Reason 1990): the nature of the task and its environmental circumstances, the mechanisms governing performance and the nature of the individual. Furthermore, the notion of intention, and the distinction between competence and control are important aspects of errors with respect to HRA (Hollnagel 1993).

An error can evolve into an accident. Accidents are the unfortunate end of a sequence of events and conditions (Hudson 2010). An important phenomenon of accidents is that in general multiple causes (or errors) can be identified (CUR 2010). Useful models of accident causation are the Domino model of Heinrich (Hudson 2010) and the 'Swiss Cheese' model of Reason (Reason 2000, Reason et al. 2001). Both models were used as a starting point for the developed model. 
Another method of error analysis is by means of Human Reliability Assessments (HRA), a specific type of Probabilistic Risk Assessments aimed at the assessment of human potential in a system. A HRA is performed by estimating the quantitative or qualitative contribution of human performance to system reliability and safety (Swain 1990, Kirwan 1994). Human error quantification is the most developed part in most HRA processes, which usually consists of calculating a Human Error Probability (HEP) (Kirwan 1994). Aspects within the working environment which affect these HEPs are: cognitive level, available time, relationship between people, work load, connections between tasks and spatial distance from task (Spurgin 2009). These factors are frequently termed performance shaping factors (DiMattia et al. 2005), and are often used in Error Management to prevent the occurrence of future human errors (Grozdanovic \& Stojiljkovic 2006). An important HEP-influencing factor is the cognitive demand level of the task. A useful distinction in these demand levels is made by Reason (1990): Skill-based, Rule-based and Knowledge-based.

Few specific HRA tools for Structural Engineering are available in the literature. An example is the research of Stewart and Melchers (Melchers 1989, Stewart 1993). Stewart's HRA model is a Monte-Carlo simulation, based on event-tree logic in which a design task is subdivided into successive individual components. A more general model is the Cognitive Reliability and Error Analysis
Method (CREAM) of Hollnagel (1998). This model is aimed towards the complex interaction between human cognition, and the situation or context in which the behaviour occurs. Stewart's model and the CREAM model are both incorporated to a certain extend in the proposed model.

\section{DEVELOPING THE HRA MODEL}

The model consists of a quantitative Human Reliability Assessment (HRA) model, for evaluating the effects of human error in structural engineering. The model starts with selecting an Engineering Process which has the potential to result in catastrophic consequences, when performed incorrectly. Through a couple of distinctive steps a relative failure probability for the specific Engineering Process is obtained. A schematic overview of this model is presented in Figure 1. The process consists of four steps, which are: identify process and boundary conditions, assess effect on basic task, assess effect on overall process and calculate failure probability of the process.

In the first step the process and its boundary conditions are identified, which is required in order to focus on the most critical processes. The second step in the model is to quantify the effect of human errors on a basic task. For this, the process is subdivided into basic tasks, for instance: consult regulations, obtain requirements, etc. Within these basic tasks, a Human Error is defined by a Human Error

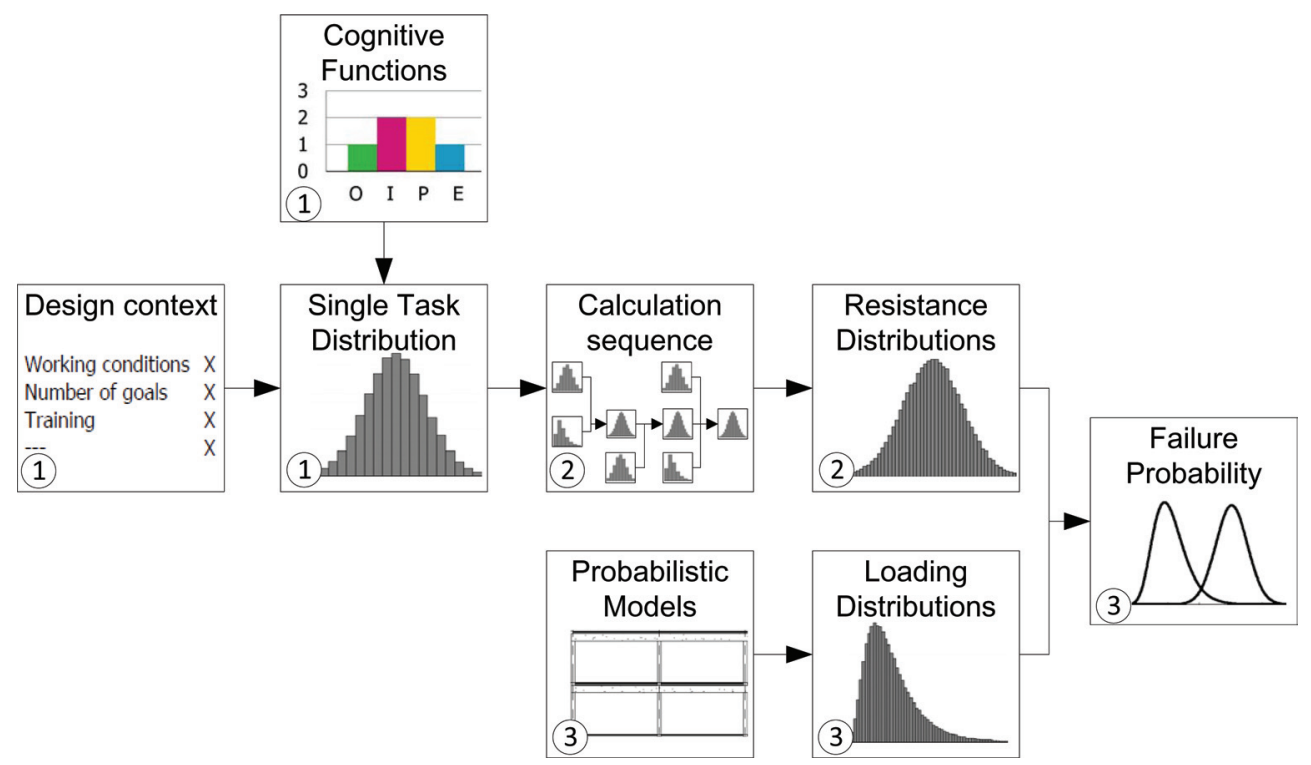

Figure 1. Simplified flow chart of the HRA model. Steps: 1) assess effect on basic task 2) assess effect on overall process 3 ) calculate failure probability of the process. 
Probability (HEP) and an Error Magnitude (EM). The HEPs are defined by evaluating the underlying cognitive functions and the particular design context of the basic task, based on the CREAM model presented in Hollnagel (1998). Within this model, each basic step is subdivided into basic cognitive tasks, for which standard failure probabilities are defined. This standard failure probability is subsequently multiplied by an adjustment factor to take the design context into account. The Error Magnitudes (EM) are defined as numerical deviations from intended values. These deviations are modelled using astandard distributions such as Normal, Lognormal, etc., which fit the characteristic of the particular task. For example the EM distribution of a calculation task consist of computation errors (Lognormal distribution) and decimal errors (Discrete distribution), as indicated by the research of Melchers (1989).

Within the structural design process typical design tasks are frequently occurring. Examples are consulting norm requirements and calculating a formula. Within this research seven basic tasks are distinguished for which a Human error distribution is calculated: Consult, Obtain, Derive, Determine, Calculate, Insert, Communicate. Furthermore, a distinction is made of the level of complexity of each task. For this, three different levels of cognitive demands are distinguished, in line with the cognitive levels suggested in Reason (1990):

- Skill-based level; highly routinised activities in familiar circumstances.

- Rule-based level; problem solving activities by means of previous established 'if-then' rules.

- Knowledge-based level; problem solving activities based on a higher level analogy.

The resulting HEP-values are shown in Table 1. In order to obtain these values an extra adjustment factor is used to take the difference between operational tasks (on which the CREAM model is based), and design tasks into account. This adjustment

Table 1. Numerical values of Human Error Probabilities (HEP).

\begin{tabular}{llll}
\hline $\begin{array}{l}\text { Basic } \\
\text { task }\end{array}$ & $\begin{array}{l}\text { Skill- } \\
\text { based }\end{array}$ & $\begin{array}{l}\text { Rule- } \\
\text { based }\end{array}$ & $\begin{array}{l}\text { Knowledge- } \\
\text { based }\end{array}$ \\
\hline Consult & $2.25 \cdot 10^{-3}$ & $1.25 \cdot 10^{-2}$ & $2.24 \cdot 10^{-2}$ \\
Obtain & $1.28 \cdot 10^{-5}$ & $2.50 \cdot 10^{-3}$ & - \\
Derive & $5.13 \cdot 10^{-4}$ & $7.63 \cdot 10^{-4}$ & $2.06 \cdot 10^{-2}$ \\
Determine & $5.13 \cdot 10^{-4}$ & $1.03 \cdot 10^{-2}$ & $3.00 \cdot 10^{-2}$ \\
Calculate & $2.56 \cdot 10^{-5}$ & $7.75 \cdot 10^{-4}$ & $2.02 \cdot 10^{-2}$ \\
Insert & $1.28 \cdot 10^{-5}$ & $2.50 \cdot 10^{-3}$ & - \\
Communicate & $7.68 \cdot 10^{-4}$ & $1.05 \cdot 10^{-3}$ & $1.10 \cdot 10^{-2}$ \\
\hline
\end{tabular}

factor $($ value $=0.5)$ is based on a comparison of the intermediate results (on the knowledge based level) with HEP values obtained for design types of tasks (Stewart 1993).

The linkage of individual tasks into an overall task is performed by means of a Monte-Carlo simulation. The procedure for simulating each basic task is based on the task-cycle approach presented in Stewart (1993). The basic task procedure is shown in Figure 2 for the typical basic task "calculate reinforcement". The procedure starts with input parameters which can be the output parameters of a preceding basic task or an input parameter from outside the considered design process. The next step is to generate a Random Number (RN) between 0 and 1 , and to obtain a Human Error Probability (HEP) for the basic task at hand. If the $\mathrm{RN}$ is smaller then the HEP, failure occurs and subsequently the output of the microtask is multiplied with an Error Magnitude (EM), obtained from the EM distribution function. If the Random Number is equal to or larger then the HEP no error occurs and subsequently the output of the micro-task is not multiplied with an Error Magnitude. The result of this procedure is an error distribution function as shown in Figure 2.

In the third step, the effects on the basic design tasks are combined into an overall effect. Which is expressed as a function of the resistance properties of the engineered system. This is implemented by linking each individual task to numerical (design) values of the considered structural design process. The basic tasks are coupled by means of an analogy based on the calculation sequence of the underlying structural design process. This methodology enables a clear relation between cause and effect, which is required from a 'chain of events' perspective.

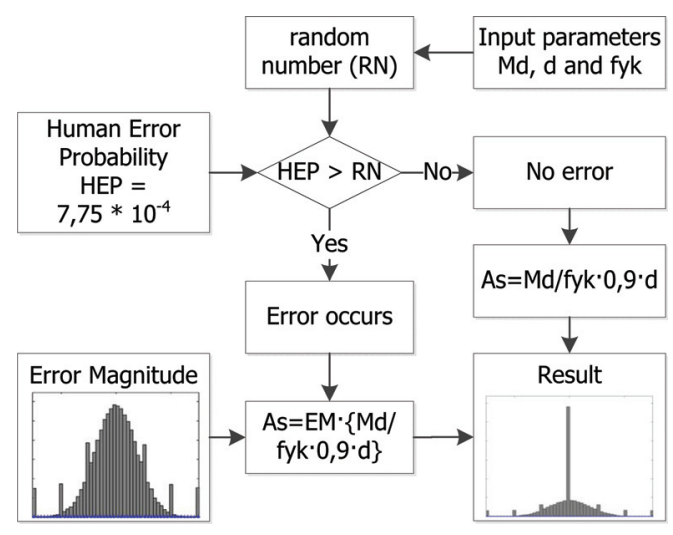

Figure 2. Monte-Carlo simulation procedure of a basic task. 


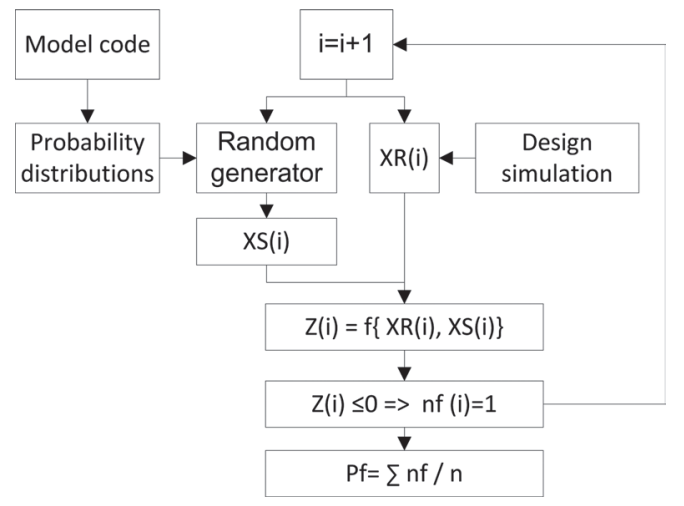

Figure 3. Monte Carlo procedure based on the reliability function.

Finally the structural failure probability is obtained by combining the resistance properties with loading conditions within a reliability analysis on element level. For this, a Monte-Carlo simulation procedure based on the reliability function of the plastic capacity of the considered element is proposed. The simulation procedure is shown in its basic form in Figure 3. The input for this simulation procedure consists of parameters obtained from the design simulation (step 3) and parameters obtained from the probabilistic model code (JCSS 2001). These input values are incorporated as deterministic values within each Monte Carlo iteration. Failure will occur if the reliability function $(\mathrm{Z}=\mathrm{R}-\mathrm{S})$ is smaller or equal to zero, in which $\mathrm{R}$ is the resistance and $\mathrm{S}$ the loading acting on structural element level. The probability of failure is subsequently estimated using the following formula:

$P_{f} \approx \frac{n_{f}}{n}$

where $n$ is the total number of simulations and $n_{f}$ is the number of simulations for which $Z \leq 0$. By running a sufficient number of simulations a reliable result is obtained.

\section{IMPLEMENTATION OF THE MODEL}

The use of the model is demonstrated by a case study, consisting of the (structural) design process of a beam element within an office building. Two beam types are distinguished: a single beam element and a frame structure as shown in Figure 4. For the simulations of the case study, the numerical calculation program Matlab is used. It should be noted that the construction process is not

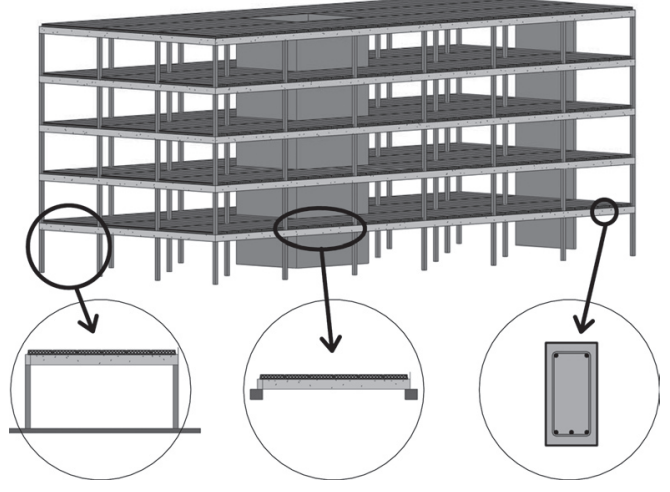

Figure 4. Overview of the office building used within the case study.

considered in this case study due to limitations in size and scope. The context of the process under consideration consists of two parts: the design object and the design organisation. The design object consists of a concrete frame structure with hollow core slabs and a stabilising core (as shown in Fig. 4). The design organization is a typical engineering company in The Netherlands.

In this case study two scenarios are considered in more detail: the level of design control by the designer/superior and the level of professional (engineering) knowledge of the structural designer. The level of professional knowledge is distinguished within the model by identifying two types of design engineers: an experienced engineer and an inexperienced engineer. For each of these two types of engineers a cognitive demand level (skill-, rule- or knowledge based) is assigned to each basic task. This results in a different error probability distribution on the basic task level, of which the effect on the total process can be assessed.

Within a design process several types of control can be distinguished. The level and profundity of control is process dependent, and differs widely. In this research, two levels of control are distinguished: Self checking (checking performed by the person who has prepared the design) and Normal Supervision (checking by different persons than those originally responsible). The level of control is incorporated in the model by using control loops within the design process. Within these loops, the process is reconsidered if the outcome is not within certain limits. Within the software of the case study, the process continues after two iterations with the incorrect value. This process is very basic but encompasses some important aspects of control: comparison of the results with an output which the designer/ supervisor deems realistic and reconsidering for a 
finite number of times if the designer/supervisor suspects in-correctness.

\section{RESULTS}

In this section, the results of the case study will be discussed, with the aim to show the potential of the model. The effect of superior/engineering control is visually demonstrated in Figure 5 by means of a histogram plot. In this plot one parameter is selected, namely the volume of top reinforcement. This is considered relevant to represent the effect of the whole process. The three bars of the histogram subsequently represent the following situations: no control sequence is used to check the results, only the designer has checked his own results based on experience and finally the designer has checked his results and a superior has checked the result as well (based on experience). It can be seen from Figure 5 that the percentage of error-free designs is increased from $78 \%$ to $85 \%$ in case of self-checking. Also the scatter within the error is reduced. In case of superior control, the figure shows that the negative errors leading to a top reinforcement lower then $1000 \mathrm{~mm}$ are almost completely disappeared. Furthermore the percentage of error-free designs is increased from $85 \%$ to $89 \%$. From this it can be concluded that selfchecking and superior control are both important aspects of human error prevention.

The effect of professional experience is visually demonstrated in Figure 6 by means of a histogram plot of the same top reinforcement. It can be seen that experience has some effect on the error-free design percentage, which is increased from $89 \%$ to $92 \%$. However one important thing to mention is that the error occurrence beneath a reinforcement area of $1500 \mathrm{~mm}$ is not decreased. This has a large

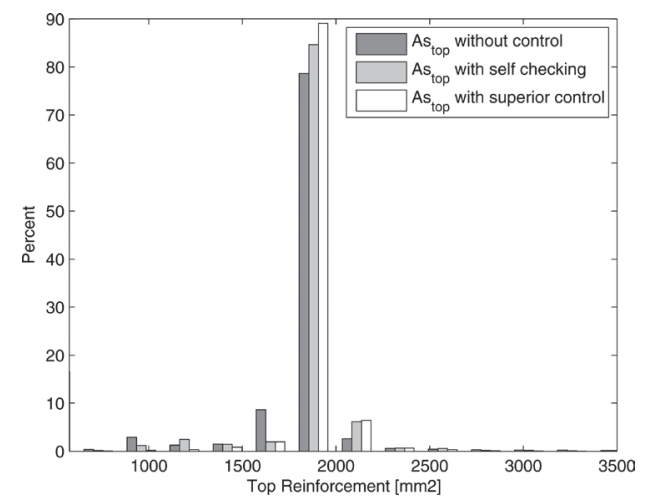

Figure 5. Function of the top reinforcement distribution depending on the level of control.

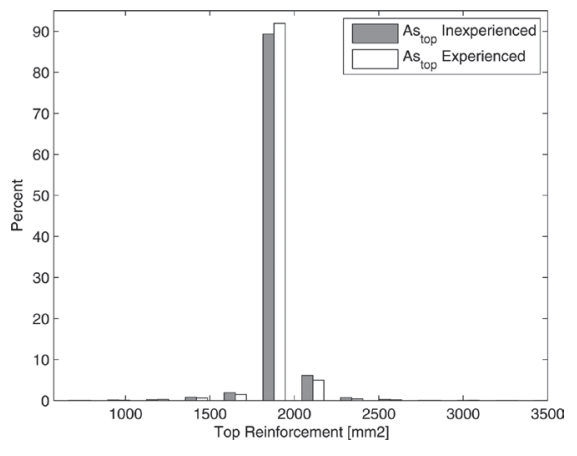

Figure 6. Function of the top reinforcement distribution depending on experience.

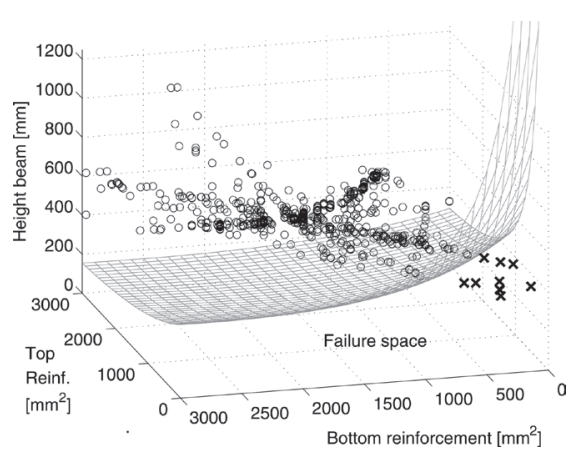

Figure 7. Solution domain of the simulation of the frame structure as a function of top/bottom reinforcement and beam height. No. of simulations: 20.000 . (Mesh) reliability function based on plastic limit state analysis. (o) Frame does not fail. (X) Frame fails.

effect on the final structural failure probability. This is due to the fact that these errors (which reduce the reinforcement area) decrease the structural resistance. From this is can be concluded that experience has also some effect, however less than the control mechanisms. This can be explained from the fact that a fairly simple design process is selected, which requires less engineering knowledge and provides sufficient control possibilities. A more complicated process will inevitable lead to other results.

Now the effect of different design sequences will be discussed. For this the results of a structural design process for a single beam (statically determinate) and a frame structure (statically indeterminate beam) is considered. The results are presented in Figures 7 and 8. The solution of a single simulation (of the Monte Carlo simulation) is presented by a circle or cross, while the mesh surface represent the reliability function. Failure will occur if the result of a simulation is beneath this 


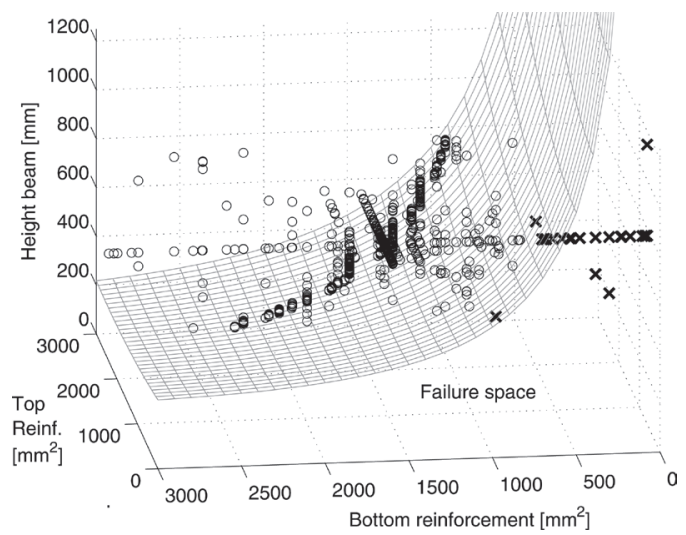

Figure 8. Solution domain of the simulation of a single beam as a function of top/bottom reinforcement and beam height. No. of simulations: 20.000. (Mesh) reliability function based on plastic limit state analysis. (०) Beam does not fail. (X) Beam fails.

mesh surface (indicated with a ' $X$ '), results above this surface are deemed safe (indicated with a $\circ^{\circ}$ ). The reliability function is considerably simplified in order to represent some characteristics of the function. Only three parameters are depicted (beam height, bottom reinforcement and top reinforcement), while the other parameters are kept fixed (as well as the loading conditions). Nevertheless both figures provide some useful insights into the results of the analysis and the used reliability function.

First of all, both reliability functions present two important aspects of failure in reinforced concrete beams: a lower reinforcement ratio results in a higher collapse probability and the reinforcement ratio has an optimum due to brittle concrete failure. However the effect of ductile behaviour, robustness and catenary effects are ignored, from which it can be concluded that the analysis on element level is insufficient to cope with system level phenomena. Secondly, when comparing both figures, the effect of different design sequences becomes visible. The scatter within the solutions in case of a frame structure is larger than in case of a single beam. However, the failure space in case of a frame structure is smaller, which results in a larger failure probability in case of a single beam element despite the smaller scatter within the beam element case. This entails that the failure probability of a structural element depends on the design process and the structural robustness of the engineered system.

Summarising all the results of the case study, the probability distributions of the simulations are presented in Table 2. The Failure probability for the single beam is higher, and the effect of engineer-
Table 2. Failure probabilities of the frame structure and single beam.

\begin{tabular}{lll}
\hline Scenario & $\begin{array}{l}\text { Frame } \\
\text { structure }\end{array}$ & $\begin{array}{l}\text { Single } \\
\text { beam }\end{array}$ \\
\hline $\begin{array}{l}\text { Experienced designer } \\
\text { with self-checking }\end{array}$ & $1.1 \cdot 10^{-3}$ & $5.2 \cdot 10^{-3}$ \\
$\begin{array}{c}\text { Experienced designer } \\
\text { with normal supervision }\end{array}$ & $0.3 \cdot 10^{-3}$ & $1.0 \cdot 10^{-3}$ \\
$\begin{array}{c}\text { Inexperienced designer } \\
\text { with self-checking }\end{array}$ & $1.3 \cdot 10^{-3}$ & $5.4 \cdot 10^{-3}$ \\
$\begin{array}{l}\text { Inexperienced designer } \\
\text { with normal supervision }\end{array}$ & $0.4 \cdot 10^{-3}$ & $1.1 \cdot 10^{-3}$ \\
\hline
\end{tabular}

ing knowledge is higher than the effect of control. These results are occurring due to the specific situation of the case study, and are not relevant for real situations. The results are only useful relative to each other, as the underlying Human Error Probabilities are quite subjective and need further calibration with real cases. As such, the model is limited to comparing different structural design processes with each other, and cannot be used to extract absolute failure probabilities.

\section{DISCUSSION}

This paper mainly focussed on the basic layout of a Human Reliability Assessment (HRA) model for structural engineering from a scientific perspective (in contrast to a practical perspective). Based on this, some usability requirements are discussed beneath. A functional requirement of the HRA model is to quantify human error in structural engineering in a realistic and useful manner. In line with (Ale et al. 2012) and (Hudson 2010) the departure point is: "[...] that control systems need to be capable of at least as much variation as the body to control" (Ale et al. 2012). This entails (among others) that the relation between an error and the causes of error should be presented on a realistic manner. Furthermore, human behaviour (Cognitive Aspects) and the (organizational) context of the situations should be taken into account.

Error causation and the influence of context are very complex to model with a HRA model due to non-linear (the causal effects are not simplified to a single chain) and non-deterministic (the relation between two values is not deterministic) behaviour of accident causation (Ale et al. 2013, Hudson 2010). Within the presented model this is incorporated to a certain extent. The non-linearity from organizational causes to structural consequences is present in the focus of the model on the complex interactions between human cognition, rather then 
defining specific routes of human information processing. The focus on cognitive failure causes rather then operational failure causes avoids the need to identify each possible cause of failure, which is rather impossible. Concerning the nondeterministic character of accident causation, the model represents the variables in terms of probability distributions rather then simple failure rates. This is in line with distributions used in (Ale et al. 2009, Ale et al. 2010). The question remains if the proposed model is advanced enough to cope with the non-linear and non-deterministic properties of accident causation. Concerning this, three aspects are identified which hamper the use of the model: factors well of the line of direct causality, common effects of higher order causes and accidents beyond imagination.

Another related issue is to what extent the model is capable to incorporate the two types of error provoking conditions (Reason 1990): active failures and latent conditions. Active failures are the unsafe acts committed, while latent conditions are unsafe conditions within a system. A HRA model should be capable to cope with both error provoking conditions in order to be reliable and realistic. Active failures are incorporated in the model by using Human Error Probabilities (HEP) and Error Magnitudes (EM) on the basic-task level to present the probability of occurrence of an active failure in the design process.

Latent conditions are incorporated by using the design context and the overall design layout. Latent conditions have large impacts on the conditions in which a design is performed. This is presented by a weighting factor depending of the design context, based on the CREAM model. Furthermore, latent conditions within the overall design process are for instance incomplete control mechanisms within the organization (which can be modelled with a design sequence). It may be questioned if all latent conditions are taken into account, and if the relation between latent condition and consequence is correct. Within building industry, many probable organizational influencing factors have been suggested (Terwel \& Vambersky 2012). However, it is very hard to detect all latent conditions, and quantify their influence. Representing this relation by means of a weighting factor and the process layout is very basic, but is a useful approximation.

\section{CONCLUSION}

This paper discussed the use of a HRA model within Structural Engineering to detect vulnerabilities within Structural Design processes as a consequence of Human Error. The model consists basically out of four steps: select process and boundary conditions, assess effects on basic tasks, assess effect on overall process and calculate failure probability. Through these steps a relative failure probability of the engineering process is obtained.

Based on the performed case study and comparison with literature it is concluded that the model has potential to identify vulnerabilities within a Structural Design process. However the model is very crude at this stage and needs further improvements to have any practical use. Furthermore, quantifying human error probabilities is sensitive to subjectivity. as a consequence, the results can only be used for relative comparison.

Due to the explorative character of this research, several opportunities for further research are identified. First of all verification and calibration of the model is required to improve the model and its practical use. Secondly, the Human Error Probabilities are primarily based on operational types of actions (in contrast to design actions considered in this research) and the Error Magnitudes within design tasks are only vaguely known. Improvements of these numerical values is required to improve the validity of the model. Another interesting aspect is the effect of learning (single loop, double loop) on the failure probabilities within the model. Also the effect of safety barriers should be considered in more detail. Safety barriers are often interrelated due to common organizational factors. Within the model this is applied on a basic manner through the amplification factor for the design context. Finally, the real behaviour of structures (robustness, ductility) at the point of failure should be incorporated and the construction stage of a building process could be investigated in more detail.

Through this case study it becomes clear that Human Reliability Assessments have the potential to be used within structural engineering. However, it still has a long way to go before HRA can be of any practical use in construction.

\section{REFERENCES}

Ale, B.J.M., L.J. Bellamy, R. Cooke, M. Duyvis, D. Kurowicka, \& P.H. Lin (2009). Causal model for air transport safety. final report. Technical report, Ministry of transport, directorate general for air transport and maritime affairs, The Hague, Netherlands.

Ale, B.J.M., L.J. Bellamy, R. van der Boom, J. Cooper, R.M. Cooke, L.H.J. Goossens, A.R. Hale, D. Kurowicka, O. Morales, A.L.C. Roelen, \& J. Spouge (2010). Further development of a causal model for air transport safety (cats): building the mathematical heart. Reliability engineering and system failure 94, 1433-1441.

Ale, B.J.M., D. Hanea, S. Sillen, P.H. Lin, C. Gulijk, \& P. Hudson (2012). Modelling risk in high hazard operations: integrating techical, organisational and cultural factors. Unpublished: Department of safety science, Delft university of technology. 
CUR (Ed.) (2010). Falende constructies. case-onderzoek naar structurele oorzaken van falen en maatregelen die dat tegengaan. Gouda: CUR Bouw en Infra. CUR publication $\mathrm{C} 232$.

DiMattia, D.G., F.I. Khan, \& P.R. Amyotte (2005). Determination of human error probabilities for offshore platform musters. Journal of Loss Prevention in the Process Industries 18, 488-501.

Ellingwood, B.R. (1987, February). Design and construction error effects on structural reliability. Journal of Structural Engineering 113(2), 409-422.

Fruhwald, E., E. Serrano, T. Toratti, A. Emilsson, \& S. Thelandersson (2007). Design of safe timber structures-how can we learn from structural failures in concrete steel and timber? Technical report, Lund Institute of Technology, Lund (Sweden).

Grozdanovic, M. \& E. Stojiljkovic (2006). Framwork for human error quantification. Philosophy, Sociology and Psychology 5(1), 131-144.

Hollnagel, E. (1993). Modelling of cognition: procedural prototypes and contextual control. Le travail humain 65(1), 27-51.

Hollnagel, E. (1998). Cognitive reliability and error analysis method. Oxford: Elsevier Science Ltd.

Hudson, P. (2010, September). Safety science: It's not rocket science, it's much harder. Repository TU Delft. received from http://repository.tudelft.nl/view/ir/uuid.

JCSS (2001). Probabilistic Model Code. Joint Committee on Structural Safety. Received from: http://www.jcss byg.dtu.dk/Publications/Probabilistic-Model-Code. aspx ISBN 978-3-909386-79-6.

Kaminetzky, D. (1991). Design and construction failures: Lessons from forensic investigations. New York: McGraw-Hill.
Kirwan, B. (1994). A guide to practical human reliability assessment. London: Taylor and Francis.

Melchers, R.E. (1989). Human error in structural design tasks. Journal of structural engineering 115(13), 1795-1807.

Reason, J.T. (1990). Human error. Cambridge: Cambridge University Press.

Reason, J.T. (2000). Managing the risks of organizational accidents. London: Ashgate.

Reason, J.T., J. Carthey, \& M.R. de Leval (2001). Diagnosing 'vulnerable system syndrome': an essential prerequisite to effective risk management. Quality in Health Care 10(Suppl II), ii21-ii25.

Spurgin, A.J. (2009). Human Reliability Assessment, theory and practice. CRC Press, Taylor and Francis Group.

Stewart, M.G. (1993). Structural reliability and error control in reinforced concrete design and construction. Structural safety 12, 277-292.

Swain, A.D. (1990). Human reliability analysis: need, status, trends and limitations. Reliability Engineering and System Safety 29, 301-313.

Terwel, K.C. \& J. Vambersky (2012). Possible critical structural safety factors: a literature review. In Forensic Engineering 2012: Gateway to a Safer Tomorrow, Conference Proceedings, pp. 408-417. ASCE.

Vrouwenvelder, A.C.W.M. (2011). Probabilistic modelling of exposure conditions for robustness. In Taller, Longer, Lighter-Meeting growing demand with limited resources, IABSE-IASS 2011 London Symposium Report, pp. 593-602. 